\title{
Some aspects of affine motion and nonholonomic constraints. Two ways to describe homogeneously deformable bodies
}

\author{
Barbara Gołubowska
}

June 25, 2018

Institute of Fundamental Technological Research, Polish Academy of Sciences $5^{B}$ Pawińskiego str., 02-106 Warsaw, Poland e-mail: bgolub@ippt.pan.pl

\begin{abstract}
This paper has been inspired by ideas presented by V. V. Kozlov in his works [19, 20. In this paper our goal is to carry out a thorough analysis of some geometric problems of the dynamics of affinely-rigid bodies. We present two ways to describe this case: the classical dynamical d'Alembert and variational, i.e., vakonomic one. So far, we can see that they give quite different results. The vakonomic model from the mathematical point of view seems to be more elegant. The similar problems were examined by Jóźwikowski and W. Respondek in their paper [16].
\end{abstract}

Keywords: affine bodies, reaction forces, vakonomic mechanics.

\section{General formulation}

The origin of the problem goes to the turn of XIX and XX centuries, or even more precisely to the second half of XIX century. Let us begin with reminding some elementary concepts. The configuration space of mechanical systems will be a differential manifold $Q$ of dimension $n$; local coordinates in $Q$, i.e., generalized coordinates will be, as usual, denoted by $q^{1}, \ldots, q^{n}$, or briefly by $q^{i}$. The manifold of positions and velocities, i.e., the tangent bundle over $Q$ will be denoted by $T Q$. It is a set-theoretical union of all tangent spaces $T_{q} Q$, i.e.,

$$
T Q=\bigcup_{q \in Q} T_{q} Q
$$

Obviously, $T_{q} Q$ is the linear space of all apriori possible velocities at $q$. Its dimension is $n$ as well, therefore $\operatorname{dim} T Q=2 n$. For any velocity vector at $q, v \in T_{q} Q$, we introduce its components $v^{i}$ with respect to generalized coordinates $q^{i}$. This induces coordinates $\left(q^{i}, v^{i}\right)$ on $T Q$. The natural projection of $T Q$ onto $Q$ will be denoted as usual:

$$
\tau_{Q}: T Q \rightarrow Q, \quad \tau_{Q}\left(T_{q} Q\right)=\{q\} .
$$

Strictly speaking, coordinates $q^{i}$ in $\left(q^{i}, v^{i}\right)$ should be rather denoted by $q^{i} \circ \tau_{Q}$, however for the obvious reasons of simplicity we use the traditional symbols $q^{i}$. Geometry of the tangent bundle is interesting in itself and may be considered as a mathematical background for the theory of the systems of second-order differential equations [1, 38, 42. Its advantage is to use only operational, directly measurable concepts like coordinates and velocities. Any curve $\rho: \mathbb{R} \rightarrow Q$ may be canonically lifted to the curve $\rho^{\prime}: \mathbb{R} \rightarrow T Q$ without introducing any additional geometry to $Q$; just $\rho^{\prime}(t)$ is the tangent vector to $\rho$ at $\rho(t)$, a uniquely defined element of the tangent space $T_{\rho(t)} Q$. And conversely, a curve $\sigma: \mathbb{R} \rightarrow T Q$ is said to be integrable if there exists a curve $\rho: \mathbb{R} \rightarrow Q$ such that $\sigma=\rho^{\prime}$. At every point $v \in T Q$ there is a manifold of integrable vectors $K_{v} \subset T_{v} T Q$ which $\tau_{Q}$ projects onto $v$,

$$
K_{v}=\left\{X \in T_{v} T Q: T \tau_{Q}(X)=v\right\} .
$$

Only integrable vectors describe accelerations of motion; this description is independent of anything like an affine connection or metric tensor in $Q$. A vector field $X: T Q \rightarrow T T Q$ on $T Q$ is said to be integrable if for any $v \in T Q$ the vector $X_{v} \in T_{v} T Q$ is integrable. This means that:

$$
T \tau_{Q} \circ X=\tau_{T Q} \circ X
$$


Obviously, $T \tau_{Q}: T T Q \rightarrow T Q$ is the tangent mapping of $T T Q$ onto $T Q$ and $X$ is defined with a mapping (cross-section) of $T Q$ into $T T Q$. This is an absolute geometric language [1, 23, 38, 42, but everything becomes clear in the local coordinate systems $\left(q^{i}, v^{i}\right)$. Then any integrable vector field is expressed locally as

$$
X=v^{i} \frac{\partial}{\partial q^{i}}+\Phi^{i}\left(q^{j}, v^{j}\right) \frac{\partial}{\partial v^{i}} .
$$

The corresponding integral curves satisfy the following local equations:

$$
\frac{d q^{i}}{d t}=v^{i}, \quad \frac{d v^{i}}{d t}=\Phi^{i}\left(q^{j}, v^{j}\right)
$$

i.e., briefly:

$$
\frac{d^{2} q^{i}}{d t^{2}}=\Phi^{i}\left(q^{j}, \frac{d q^{j}}{d t}\right)
$$

This is the general form of Newtonian equations. It is seen that this structure is inherent in the tangent bundle $T Q$. Nevertheless, it is too poor for developing the details of both unconstrained and constrained dynamics. Basing merely on those elements we are unable to define such important concepts like energy, power, and work, necessary for dealing with constraints in either d'Alembert or vakonomic sense. The missing elements are those in a sense contained in another concept of the state space, namely the cotangent bundle $T^{*} Q$, i.e., the set-theoretical union of all spaces $T_{q}^{*} Q$ dual to $T_{q} Q$,

$$
T^{*} Q=\bigcup_{q \in Q} T_{q}^{*} Q
$$

The natural projection from this bundle onto $Q$ will be denoted by $\tau_{Q}^{*}$,

$$
\tau_{Q}^{*}: T^{*} Q \rightarrow Q, \quad \tau_{Q}^{*}\left(T_{q}^{*} Q\right)=\{q\}
$$

This cotangent bundle, i.e., Hamiltonian state space of the system, dual to the Newton state space $T Q$, is endowed with the canonical symplectic geometry [1, 23, 38, based on the two-form:

$$
\gamma=d \theta
$$

where $\theta$ is the canonical Cartan differential one-form:

$$
\theta_{p}:=p \circ T \tau_{Q_{p}}^{*}
$$

Introducing components $p_{i}$ of $p$ we obtain coordinate systems $\left(q^{i}, p_{i}\right)$ defined locally on $T^{*} Q$. It is well known and can be easily checked that locally

$$
\theta=p_{i} d q^{i}, \quad \gamma=d \theta=d p_{i} \wedge d q^{i}
$$

This is another version of the state space. Its peculiarity is the existence of the tensor fields (12). Unlike this, there is no natural lift of curves from $Q$ to $T^{*} Q$, and the elements of fibres, i.e., canonical momenta $p \in T^{*} Q$ are not operationally interpretable. Nevertheless, it is a peculiarity of analytical mechanics, especially with constraints, that the both state spaces $T Q$ and $T^{*} Q$, i.e., the $\mu$-phase space and the usual (Hamiltonian) state space, must be used. The correspondence between them is based on the use of Lagrangian or Hamiltonian. Namely, the Lagrange function $L: T Q \rightarrow \mathbb{R}$ gives rise to the Legendre transformation $\mathcal{L}: T Q \rightarrow T^{*} Q$, namely

$$
\mathcal{L}(v):=D_{v}\left(L \mid T_{q} Q\right), \quad v \in T_{q} Q .
$$

Analytically:

$$
\mathcal{L}:\left(q^{i}, v^{i}\right) \rightarrow\left(q^{i}, p_{i}\right)=\left(q^{i}, \frac{\partial L}{\partial v^{i}}\right)
$$

The energy function $E: T Q \rightarrow \mathbb{R}$ is given by:

$$
E(v)=\langle\mathcal{L}(v), v\rangle-L(v)=v^{i} \frac{\partial L}{\partial v^{i}}-L(v) .
$$

If $\mathcal{L}$ is invertible, the necessary although non-sufficient condition for this is that the Hessian of $L$ is non-vanishing,

$$
\operatorname{det}\left[\frac{\partial^{2} L}{\partial v^{i} \partial v^{j}}\right] \neq 0
$$


then $E$ gives rise to the Hamilton function $H: T^{*} Q \rightarrow \mathbb{R}$, namely:

$$
E=H \circ \mathcal{L}, \quad H=E \circ \mathcal{L}^{-1} .
$$

In this paper it is always assumed.

Let us only mention incidentally that if $\mathcal{L}(T Q)=M \subset T^{*} Q$, the Hamilton function is still defined, but only on $M$, or more rigorously, on the connected components of $M$. Although this situation is also physically interesting, we do not consider it here [1, 42. Let us mention finally, that in the hyper-regular case, when $\mathcal{L}$ is invertible, the inverse mapping is analytically given by the following expression analogous to (14):

$$
\mathcal{L}^{-1}:\left(q^{i}, p_{i}\right) \rightarrow\left(q^{i}, v^{i}\right)=\left(q^{i}, \frac{\partial H}{\partial p_{i}}\right) .
$$

The variational principle for the Lagrangian dynamical system,

$$
\delta \int L d t=0
$$

leads to the usual Lagrange equations of the second kind:

$$
-\frac{\delta L}{\delta q^{i}(t)}=\frac{d}{d t} \frac{\partial L}{\partial \dot{q}^{i}}-\frac{\partial L}{\partial q^{i}}=0, \quad i=1, \ldots, n .
$$

If in addition to variational forces derivable from $L$ also some other, first of all dissipative ones $D_{i}$, are present, then equations (20) are replaced by:

$$
\frac{d}{d t} \frac{\partial L}{\partial \dot{q}^{i}}-\frac{\partial L}{\partial q^{i}}=D_{i}
$$

Let us stress that $D_{i}$ geometrically is a covector field. The energy balance has the form:

$$
\frac{d E}{d t}=-\frac{\partial L}{\partial t}+D_{i} \frac{d q^{i}}{d t}
$$

The non-conservation of $E$ follows from two circumstances:

- 1) the explicit dependence of $L$ on $t$ (in addition to usual dependence of $q^{i}, \dot{q}^{i}$ on $t$ ),

- 2) the power of non-variational forces equals $D_{i}\left(d q^{i} / d t\right) ; D_{i}$ is a covariant vector.

This was description of unconstrained systems. Let us now assume that the system is subject to holonomic constraints which restrict its motion to some submanifold $W \subset Q$. One assumes then that also the tangent bundle, i.e., manifold of Newtonian states is restricted from $T Q$ to $T W$. Nevertheless, some qualitative comments are necessary here. First of all: what does it mean that the motion is restricted to $W$ and what is the status of resulting equations of motion? First of all let us assume some metric tensor $g$ in $Q$ and some field of forces which on $W$ is tangent to it. They may be encoded in the expression for the total potential energy. Let $W$ be given by equations

$$
F_{a}\left(q^{1}, \ldots, q^{n}\right)=0, \quad a=1, \ldots, m
$$

where

$$
d F_{1}(q) \wedge d F_{2}(q) \wedge \ldots \wedge d F_{a}(q) \neq 0
$$

for any $q \in W$, i.e., satisfying (23). We assume that the potential of forces $g$-orthonormal to $W$ is given by

$$
U_{\kappa}=\frac{1}{2} \kappa^{a b} F_{a} F_{b}
$$

and the total potential energy has the form:

$$
U=U_{\kappa}+V
$$

where $V$ is independent on the quantity $\kappa$.

The coefficients $\kappa^{a b}$ are assumed to be sufficiently large to force the system to remain permanently in a small neighbourhood of $W$. Equations of motion following from the Lagrangian $L=T-U$ are dependent on the parameters $\kappa^{a b}$ and one can perform the limit transition $\kappa^{a b} \rightarrow \infty$. One can show 2 that in the limit, with initial conditions compatible with $W$, one obtains that the trajectory is constrained in $W$ and satisfies the following equations:

$$
\frac{d}{d t} \frac{\partial L^{W}}{\partial \dot{x}^{\mu}}-\frac{\partial L^{W}}{\partial x^{\mu}}=0, \quad \mu=1, \ldots,(n-m)
$$


where $L^{W}$ is the restriction of $L: T Q \rightarrow \mathbb{R}$ to the tangent submanifold $T W \subset T Q$. The quantities $x^{\mu}$ are local coordinates (parameters) on $W ; q^{i}$ are their functions:

$$
q^{i}=f^{i}\left(x^{\mu}\right)
$$

Obviously, we have

$$
L^{W}(x, \dot{x}):=L\left(f^{i}(x), \frac{\partial f^{j}}{\partial x^{\mu}} \dot{x}^{\mu}\right) .
$$

One can show that the formula similar to (21), (27) is also satisfied when dissipative forces are present:

$$
\frac{d}{d t} \frac{\partial L^{W}}{\partial \dot{x}^{\mu}}-\frac{\partial L^{W}}{\partial x^{\mu}}=D_{\mu}^{W}
$$

where the covariant dissipative force on $W$ is given by

$$
D_{\mu}^{W}(x, \dot{x})=D_{i}\left(f^{j}(x), \frac{\partial f^{l}}{\partial x^{\lambda}} \dot{x}^{\lambda}\right) \frac{\partial f^{i}}{\partial x^{\mu}} .
$$

To discuss and understand properly the peculiarity of vakonomic systems it is however more natural to remain on the level of the implicite description. Namely, when dealing with a holonomically constrained dynamical system, the principle of the action minimum (or in any case, stationary point) (19) is replaced by that of conditional, i.e., constrained extremum (stationary point):

$$
\delta \int L d t=0, \quad F_{a}(q)=0, \quad a=1, \ldots, m
$$

It claims that realistic motions are those for which the variational condition in (32) is satisfied for those virtual motions which are compatible with the second subsystem. But, according to the Lusternik theorem about conditional extrema, they are those which give stationary value to the functional:

$$
\int\left(L+\mu^{a} F_{a}\right) d t=\int L^{\prime} d t
$$

where $\mu^{a}$ are apriori non-specified Lagrange multipliers, in a sense additional state variables. Performing the variational procedure for all variables we obtain:

$$
\frac{d}{d t} \frac{\partial L}{\partial \dot{q}^{i}}-\frac{\partial L}{\partial q^{i}}=\mu^{a} \frac{\partial F_{a}}{\partial q^{i}}, \quad F_{a}(q)=0 .
$$

And similarly for systems with dissipative forces we obtain:

$$
\frac{d}{d t} \frac{\partial L}{\partial \dot{q}^{i}}-\frac{\partial L}{\partial q^{i}}=D_{i}+R_{i}=D_{i}+\mu^{a} \frac{\partial F_{a}}{\partial q^{i}}, \quad F_{a}(q)=0
$$

The quantities $R_{i}$ given by

$$
R_{i}=\mu^{a} \frac{\partial F_{a}}{\partial q^{i}}
$$

are reaction forces responsible for maintaining the constraints given by (23). The total system (35) is imposed on the time-dependence of the $(n+m)$ quantities $q^{i}, \mu^{a}$.

Let us notice some essential point. Reaction forces $R_{i}$ are defined and (in general) non-vanishing on the manifolds of constrained motion $W \subset Q, T W \subset T Q$. Unlike this, the physical reactions of approximately constrained motion exactly vanish on $W$ and in a small neighbourhood of $W$ they are very large and act attractively towards $W$. Their potential is just $U_{\kappa}(25)$, (26). Because of this, for any finite $\kappa^{a b}$ the system performs quick oscillations about $W$, with a very small amplitude. This difference between physical reactions for finite $\kappa^{a b}$ and the ideal reactions (36) is very essential when the quantized problem is investigated. Namely, the mentioned small oscillations about $W$ are then labelled by large quantum numbers.

\section{Quantization}

The problem becomes complicated and in certain situations the results may by qualitatively different from those obtained by the intrinsic quantization in $W$. We do not get into details here. We only mention what is meant here by the "intrinsic quantization". Let the metric tensor on $W$ induced by the restriction of $g$ to $W$ be denoted by $g^{W}$, just the pull-back of $g$,

$$
g^{W}=\imath^{*} \cdot g,
$$


where $\imath: W \rightarrow Q$ is the natural injection, and let the effective potential energy on $W$ be denoted by $V^{W}$,

$$
V^{W}=\imath^{*} \cdot V=V \circ \imath .
$$

Then the intrinsic $W$-quantization is based on the Hamilton operator:

$$
\mathbb{H}=-\frac{\hbar^{2}}{2 m} \Delta\left[g^{W}\right]+V^{W},
$$

where $\Delta\left[g^{W}\right]$ is the Laplace-Beltrami operator acting on wave functions on $W$,

$$
\Delta\left[g^{W}\right] \Psi=\frac{1}{\sqrt{\left|g^{W}\right|}} \sum_{\mu, \nu} \frac{\partial}{\partial x^{\mu}}\left(\sqrt{\left|g^{W}\right|} g^{W \mu \nu} \frac{\partial \Psi}{\partial x^{\nu}}\right),
$$

and obviously

$$
\left|g^{W}\right|=\sqrt{\left|\operatorname{det}\left[g_{\mu \nu}^{W}\right]\right|} .
$$

It is clear that the quantum numbers of $\mathbb{H}$ may happen to interfere badly with those of the $U_{\kappa}$-terms (25), (26) for large but finite $\kappa$. This may be interesting in quantum studies of the constrained motion, and really it seems that there are some doubful problems there. However, here we do not deal with those problems there. In any case it seems that there are two not necessarilly equivalent approaches: one based on the formal restriction of the metric to constraints, and another, based on the properties of the potential. In any case even here there are some indications that the d'Alembert procedure need not by convincing.

\section{Description of dynamics by d'Alembert and vakonomic theories}

Let us go back to classical problems. It is clear that the reactions (36) do no work along any curve compatible with constraints, their power vanishes then:

$$
R_{i} \frac{d q^{i}}{d t}=\mu^{a} \frac{\partial F_{a}}{\partial q^{i}} \frac{d q^{i}}{d t}=0
$$

if for any $t, q^{i}(t) \in W$; this is a direct consequence of (32). In classical textbooks it is formulated as the principle of vanishing work along any trajectory compatible with constraints,

$$
R_{i} \delta q^{i}=\mu^{a} \frac{\partial F_{a}}{\partial q^{i}} \delta q^{i}=0
$$

if $\delta q^{i}$ is tangent to $W$, i.e., if

$$
\frac{\partial F_{a}}{\partial q^{i}} \delta q^{i}=0
$$

quite independently of any dynamical equations. In XIX-XX-th centuries the analysis of virtual displacements was treated very seriously and some art concerning the constitutive relations for them was developed. Let us remind the Appell-Chetaev, Gauss and other treatments. They were motivated by non-holonomic constrains, in particular for ones non-linear in velocities. Some of them were very inventive, although there are also opinions that certain artefacts and misconceptions appeared in them [24, 38. The point is that for Lusternik procedures one obtains different, non-convincing results.

Let us assume that the system is subject to some purely non-holonomic constraints $M \subset T Q$ linear in velocities. This means that $\tau_{Q}(M)=Q$ and for any $q \in Q$ the manifold $M_{q}:=M \cap T_{q} Q$ is a linear $(n-m)$ dimensional subspace. One can also admit it to be an affine subspace of $T_{q} Q$, i.e., some translation of a linear subspace by a vector non-contained in $M_{q}$. However, for simplicity we do not consider this modification here. Therefore, $M$ has equations of the form:

$$
F_{a}(q, v)=\omega_{a i}(q) v^{i}=0, \quad a=1, \ldots, m .
$$

We assume also that the constraints are not semi-holonomic, i.e., that the Pfaff systems

$$
\omega_{a}=\omega_{a i}(q) d q^{i}=0
$$

is not maximally integrable. If it was, the manifold $Q$ would be foliated by the $m$-dimensional family of mutually disjoint $(n-m)$-dimensional integral surfaces of (45). This would be the case if the following condition was satisfied:

$$
d \omega_{a} \wedge \omega_{1} \wedge \ldots \wedge \omega_{m}=0, \quad a=1, \ldots, m .
$$


So, we assume that the problem is purely non-holonomic, does not reduce to the family of holonomic constraints, and therefore, the family of configurations which may be reached from any point of $Q$ is $n$-dimensional. The statement "may be reached" is meant here in a purely geometric, non-dynamical sense: just reached by any integral curve of (45), without any dynamical assumption. Let us again apply the Lusternik theorem to the constrained variational problem:

$$
\delta \int L(q(t), \dot{q}(t)) d t=0, \quad F_{a}(q(t), \dot{q}(t))=\omega_{a i}(q) \frac{d q^{i}}{d t}=0
$$

So, we look for the extremals (more precisely-stationary points) of the functional

$$
[q(\cdot)] \rightarrow \int L(q(t), \dot{q}(t)) d t
$$

on the family of curves satisfying the second condition of (47). But this means that again we introduce additional parameters $\mu^{a}, a=1, \ldots, m$, i.e., Lagrange multipliers, and look for the family of unconstrained dynamical systems for $t \rightarrow\left(q^{i}(t), \mu^{a}(t)\right)$ described by:

$$
\delta \int \widetilde{L}(q(t), \dot{q}(t) ; \mu(t)) d t=0
$$

where $\widetilde{L}$ is given by:

$$
\widetilde{L}(q(t), \dot{q}(t) ; \mu(t))=L(q(t), \dot{q}(t))+\mu^{a}(t) F_{a}(q(t), \dot{q}(t)) .
$$

Performing carefully the unconstrained variational procedure for $(q(t), \mu(t))$, we finally obtain the system:

$$
\frac{d}{d t} \frac{\partial L}{\partial \dot{q}^{i}}-\frac{\partial L}{\partial q^{i}}=R_{i}, \quad \omega_{a i}(q) \frac{d q^{i}}{d t}=0
$$

where

$$
R_{i}=-\frac{d \mu^{a}}{d t} \omega_{a i}+\mu^{a}\left(\frac{\partial \omega_{a j}}{\partial q^{i}}-\frac{\partial \omega_{a i}}{\partial q^{j}}\right) \frac{d q^{j}}{d t} .
$$

Similarly, when non-variational interactions are admitted, we have:

$$
\frac{d}{d t} \frac{\partial L}{\partial \dot{q}^{i}}-\frac{\partial L}{\partial q^{i}}=D_{i}+R_{i}
$$

where $R_{i}$ is as above in (52) and $D_{i}$ are covariant components of forces non-derivable from Lagrangian. It is seen that the structure of $R_{i}$ differs form (36) by the "magnetic-like" second term of (52). But nevertheless one can think just like in holonomic systems and assume that reactions do no work on virtual displacements compatible with constraints. Therefore, they are power-free on velocities satisfying second equation of (51), i.e.,

$$
R_{i} \frac{d q^{i}}{d t}=0, \quad \text { if } \quad \omega_{a i} \frac{d q^{i}}{d t}=0 .
$$

Then

Therefore, again we would have

$$
R_{i}=\lambda^{a} \omega_{a i}=-\frac{d \mu^{a}}{d t} \omega_{a i}
$$

$$
\frac{d}{d t} \frac{\partial L}{\partial \dot{q}^{i}}-\frac{\partial L}{\partial q^{i}}=D_{i}+\lambda^{a} \omega_{a i}
$$

just like in (35), (36), this time with $\omega_{a i}$ instead of $\partial F_{a} / \partial q^{i}$. Moreover, it turns out that (56) is not only geometrically possible but just physically correct for all known problems of sliding-free rolling on rough surfaces. But mathematically (56) are non-variational in structure. An explanation was that the corresponding reactions arise through the non-variational friction phenomena [24].

Nevertheless the variational equations (51)-(53) with their intriguing "magnetic-like" correction terms seemed to be so exciting that they were intensively studied even for the purely mathematical/geometrical purposes. Various names were used for them like vakonomy (from "variational axiomatic kind").

It is clear that the second term in (52) does not influence the energy balance of reactions,

$$
R_{i} \frac{d q^{i}}{d t}=-\frac{d \mu^{a}}{d t} \omega_{a i} \frac{d q^{i}}{d t}
$$

It is admissible due to its very "magnetic" structure. In the period when the analysis of various definitions of "virtual displacements" was fashionable, there were some attempts to modify in a non-variational way the 
mutual ratio of coefficients at the two terms of (52). But the violation of the variational character of (52) seems to be the price paid for nothing. Besides, in non-holonomic systems the reaction of the classical expression for the virtual displacement,

$$
\omega_{a i}(q) \delta q^{i}=0
$$

although physically correct in the rolling case, seems to be frustrating. Indeed, when the higher-order terms are neglected, then

$$
\delta F_{a}=F_{a}(q+\delta q)-F_{a}(q)=\frac{d}{d t}\left(\omega_{a i} \frac{d q^{i}}{d t}\right)+\left(\omega_{a j, i}-\omega_{a i, j}\right) \frac{d q^{j}}{d t} \delta q^{i} .
$$

But (58) does not imply $\delta F_{a}$ to vanish. Instead, the two terms in (59) should separately vanish. But those $2 m$ conditions would be too much. The only possibility to remain within variational scheme is just to take only $m$ conditions:

$$
\frac{d}{d t}\left(\omega_{a i} \dot{q}^{i}\right)+\left(\omega_{a j, i}-\omega_{a i, j}\right) \dot{q}^{j} \delta q^{i}=0
$$

Yes, but as said above, in the physical case of sliding-free rolling, one uses (56), (58). What would be the domain of applications of incomparatively more elegant vakonomic systems? First of all, let us notice that the VAK-dynamics is very interesting from the purely mathematical point of view and that it has given rise to the completely new domain of mathematical physics based on variational principles [3, 8, 38. What concerns doubtful practical applications of vakonomy, they are in financial dynamical problems. And it is not clear if they should not be used in molecular and nuclear dynamics [38. But what is very important, it seems that the vakonomic ideas may be very useful in certain problems of the control theory. We mean here the active control procedures, not the friction-based methods. There is a feeling that the active problems are much more effective and natural due to their invariance properties. And besides, other methods are completely non-useful in nonlinear and differential higher-order problems.

Let us begin with the nonlinear case. We assume that the manifold of admissible virtual velocities at the configuration $q \in Q$ is given by the set $V_{q}$. For simplicity we assume that at any $q \in Q$ all the manifolds $V_{q}$ have the same dimension $(n-m)$. Therefore, the total manifold of constraints $M$,

$$
M=\bigcup_{q \in Q} V_{q},
$$

is a geometric plane given by equations:

$$
F_{a}\left(q^{1}, \ldots, q^{n} ; v^{1}, \ldots, v^{n}\right)=0, \quad a=1, \ldots, m .
$$

For simplicity we assume that the range of positions $\left(q^{1}, \ldots, q^{n}\right)$ is non-restricted and it is really the range of velocity variables that is subject to constraints. Therefore,

$$
\operatorname{Rank}\left[\frac{\partial F_{a}}{\partial v^{i}}\right]=n-m \text {. }
$$

When the constraints (61), (62) are nonlinear, then the procedure of eliminating constraints with the help of the above-quoted procedures is literally meaningless. Indeed, in general the principle of virtual displacements does not allow us to formulate true equations of motion. In fact, when $V_{q}$ is a general differential sub-manifold of $T_{q} Q$, then the linear shell (closure) of $V_{q}$-elements will have higher dimension than $V_{q}$ and in particular, it may coincide with $T_{q} Q$ itself. There were many attempts to prevent this. In a sense, the simplest and most general of them was the Appell-Chetaev procedure [15, 24. Let us quote the general ideas of that methods. Namely, we can try to go back to the philosophy of virtual displacements and define them as ones satisfying:

$$
\frac{\partial F_{a}}{\partial \dot{q}^{i}} \delta q^{i}=0, \quad a=1, \ldots, m,
$$

again with the summation convention applied. This is equivalent to

$$
R(q, v)_{i}=\lambda^{a} \frac{\partial F_{a}}{\partial v^{i}}
$$

for reaction forces. This follows from the fact that for any $q \in Q, v \in V_{q}$, and $w \in T_{v} M_{q} \subset T_{q} Q$ the following equation holds:

$$
\langle R(q, v), w\rangle=R(q, v)_{i} w^{i}=0 .
$$

Therefore, equations of motion may be written in the variational form:

$$
\left(\frac{d}{d t} \frac{\partial L}{\partial \dot{q}^{i}}-\frac{\partial L}{\partial q^{i}}\right) \delta q^{i}=0, \quad F_{a}(q, \dot{q})=0, \quad \frac{\partial F_{a}}{\partial \dot{q}^{i}} \delta q^{i}=0,
$$


or in the following explicit form:

$$
\frac{d}{d t} \frac{\partial L}{\partial \dot{q}^{i}}-\frac{\partial L}{\partial q^{i}}=D_{i}+\lambda^{a} \frac{\partial F_{a}}{\partial \dot{q}^{i}}, \quad F_{a}(q, \dot{q})=0
$$

These equations (68) must be jointly solved for the time dependence of $\left(q^{i}, \lambda^{a}\right)$, in general without the possibility of separating $\lambda^{a}$ and substituting them to the equations for $q^{i}$.

Let us stress that the above procedure defining $\delta q^{i}$ is mathematically correct and geometric, although in general physically non-convincing. It does not seem to refer to the energy balance or mechanical work. It also fails to describe the tangent vector in the functional manifold of motions compatible with constrains. In general, the XIX-th century procedure for establishing some explicit rules for virtual displacements, in spite of its inventive power, seems to be rather misleading from the point of view of the active control problems of servomechanisms. In particular, this concerns the problems of motion of satellites and other space-moving objects. It seems that the d'Alembert procedure and the acceleration-dependent Gauss method are definitely less reliable in such problems.

This seems particularly hopeful when we are looking for the program forces which keep velocity constant or appropriately programmed, both in the sense of direction or magnitude. Let us consider a general mechanical system with nonlinear non-holonomic constraints. We begin with first-order differential constraints, i.e., ones imposed on configurations and generalized velocities, but now without any assumption of linearity. Let us begin with the variational principle

$$
\delta \int L(q(t), \dot{q}(t)) d t=0
$$

with extra imposed nonlinear constraints:

$$
F_{a}(q, \dot{q})=0, \quad a=1, \ldots, m .
$$

The resulting equations of motion have the form following from the Lusternik principle:

$$
\frac{d}{d t} \frac{\partial L}{\partial \dot{q}^{i}}-\frac{\partial L}{\partial q^{i}}=R_{i}, \quad F_{a}(q, \dot{q})=0
$$

where the variational reactions are given by:

$$
R_{i}=\lambda^{a} \frac{\partial F_{a}}{\partial q^{i}}-\frac{d}{d t}\left(\lambda^{a} \frac{\partial F_{a}}{\partial \dot{q}^{i}}\right)=-\frac{d \lambda^{a}}{d t} \frac{\partial F_{a}}{\partial \dot{q}^{i}}+\lambda^{a} \frac{\partial F_{a}}{\partial q^{i}}-\lambda^{a} \frac{\partial^{2} F_{a}}{\partial \dot{q}^{i} \partial \dot{q}^{j}} \frac{d^{2} q^{j}}{d t^{2}}-\lambda^{a} \frac{\partial^{2} F_{a}}{\partial \dot{q}^{i} \partial q^{j}} \frac{d q^{j}}{d t} .
$$

When dissipative forces are present, then (171) becomes:

$$
\frac{d}{d t} \frac{\partial L}{\partial \dot{q}^{i}}-\frac{\partial L}{\partial q^{i}}=D_{i}+R_{i}, \quad F_{a}(q, \dot{q})=0 .
$$

Let us observe that now the quantities $\left(q^{i}, \lambda^{a}\right)$ occur in equations of motion on the almost equal footing as a kind of generalized coordinates. Because of this now there is really no chance for elimination of reactions $\lambda^{a}$ from our equations. They must be found simultaneously from those equations.

It is clear that $R_{i}$ in (72) contains also the Appell-Chetaev term

$$
-\frac{d \lambda^{a}}{d t} \frac{\partial F_{a}}{\partial \dot{q}^{i}},
$$

as mentioned above, geometrically well defined. Nevertheless, there are also three other terms, much more interesting from the point of view of the active control. For example, we may be interested in fixing with the help of some constraints the absolute value of velocity of some space-moving object. It is clear that the d'Alembert procedure developed for non-holonomic constraints with equations linear in velocities rather completely fails when dealing with such problems. Although, one must say that there were some attempts of using them, based on a strange procedure of the limit transition connected with the reduction of the number of degrees of freedom. But they were non-convincing and subject to certain criticism [24]. In any case it seems obvious that the procedure of the friction-based explanation of control seems to be exotic there. Let us compare the two procedures. We assume the simplest Lagrangian of the from:

$$
L=\frac{m}{2} g_{i j} \dot{q}^{i} \dot{q}^{j}-V(q)
$$

where the metric tensor $g_{i j}$ is assumed to have constant coefficients (so, it is curvature-free). Nonlinear constraints fix the absolute value of velocity,

$$
F(\dot{q})=\underset{8}{g_{i j} \dot{q}^{i} \dot{q}^{j}-c^{2},}
$$


where $c$ is a constant. The resulting Appell-Chetaev leads to the following system of equations:

$$
m \frac{d^{2} q^{i}}{d t^{2}}-\lambda \frac{d q^{i}}{d t}+g^{i j} \frac{\partial V}{\partial q^{j}}=0, \quad g_{i j} \frac{d q^{i}}{d t} \frac{d q^{j}}{d t}-c^{2}=0 .
$$

Unlike this, the variational vakonomic procedure gives us:

$$
(m+\lambda) \frac{d^{2} q^{i}}{d t^{2}}-\frac{d \lambda}{d t} \frac{d q^{i}}{d t}+g^{i j} \frac{\partial V}{\partial q^{j}}=0, \quad g_{i j} \frac{d q^{i}}{d t} \frac{d q^{j}}{d t}-c^{2}=0 .
$$

It is clear that the both systems are different and non-equivalent. Again in the vakonomic Lusternik procedure $\lambda$ is a kind of degree of freedom subject jointly with $q^{i}$ to a system of differential equations. And really it seems more natural here to use $\lambda$ as the active control factor. Besides, it influences the inertial properties (mass) of the body by introducing the additional, dynamical mass term $\lambda\left(d^{2} q^{i} / d t^{2}\right)$.

It is interesting to interpret physically some terms of (172). The third term may be interpreted as a channel which controls the inertia, i.e., the effective mass of the body. The resulting control may regulate, e.g., stabilize the angular velocity of rotors. The fourth term seems to influence the damping and acceleration forces, and also some gyroscopic or magnetic-like behavior, especially when combined appropriately with the second term.

Let us stress that we are thinking here about the problems of the active control. This brings about the question if our reaction are adiabatic, i.e., if they do no work. As expected, it turns out that in general it is not the case. Namely, one can show that the power of vakonomic reactions need not vanish. Indeed,

$$
R_{i} \frac{d q^{i}}{d t}=\lambda^{a} \frac{d F_{a}}{d t}-\frac{d}{d t}\left(\lambda^{a} \frac{\partial F_{a}}{\partial \dot{q}^{i}} \dot{q}^{i}\right)
$$

Obviously, the first term vanishes in a consequence of the very equations of constraints. Therefore,

$$
R_{i} \dot{q}^{i}=-\frac{d}{d t}\left(\lambda^{a} \frac{\partial F_{a}}{\partial \dot{q}^{i}} \dot{q}^{i}\right) .
$$

And in general case this is non-vanishing. But it is interesting to ask for the special case when that term does vanish. It turns out that it happens when constraints are linear in velocities. Indeed, linear functions are homogeneous of degree one, i.e., the following holds for them:

$$
v^{i} \frac{\partial F_{a}}{\partial v^{i}}=F_{a} .
$$

Therefore, (80) becomes:

$$
R_{i} \dot{q}^{i}=-\frac{d}{d t}\left(\lambda^{a} F_{a}\right)
$$

This expression evidently vanishes along any curve compatible with the imposed constraints. It is even more, namely (82) vanishes when functions $F_{a}$ satisfy the following differential equation:

$$
v^{i} \frac{\partial F_{a}}{\partial v^{i}}=F_{a}
$$

which implies that (80) becomes

$$
\frac{d}{d t}\left(\lambda^{a} F_{a}\right)=0
$$

along any curve compatible with constraints. Moreover, expression (80) vanishes also if the following holds:

$$
\left.v^{i} \frac{\partial F_{a}}{\partial v^{i}}\right|_{M}=0
$$

or, equivalently if for some functions $g_{a}^{b}$ well defined in a neighbourhood of $M$ the equation

$$
v^{i} \frac{\partial F_{a}}{\partial v^{i}}=g_{a}^{b} F_{b}
$$

is satisfied. But those facts imply that the field of vectors $v^{i}\left(\partial / \partial v^{i}\right)$ is tangent to $M$. However, it is clear that this vector field is a generator of the group of dilatations, namely $v \rightarrow e^{\tau} v$ in tangent spaces of $Q$. Therefore, its integral curves are half-spaces of one-dimensional subspaces of the tangent spaces $T_{q} Q$. Or, in the singular case, they are simply null elements of $T_{q} Q$. This means that every $M$-manifold satisfying (86) is built of onedimensional subspaces of the tangent spaces $T_{q} Q$. And every one-dimensional subspace, or rather half-subspace, 
is either contained in $M$ or just disjoint with $M$. Therefore, the manifold $M_{q}=M \cap T_{q} Q$ induces constraints on the directions in $T_{q} Q$, but not on the magnitudes of vectors.

This implies an important feature of non-holonomic constraints: if non-holonomic conditions $M$ restrict the manifold of directions of velocities, but do not influence their magnitudes, then the Lusternik reactions are adiabatic, i.e., they do no work. This result is interesting and expected. It is difficult to realize the control, and in particular stabilization of velocity, on the basis of the d'Alembert procedure, without using the energy income to the system. Unlike this, it is possible when we wish to keep or control the direction of motion.

Following (80) we can write the variational Lusternik energy balance as follows:

$$
\frac{d}{d t} E[L, M]=\frac{d}{d t}\left(E[L]+\lambda^{a} \frac{\partial F_{a}}{\partial \dot{q}} \dot{q}^{i}\right)=D_{i} \dot{q}^{i}
$$

Here we have obviously:

$$
E[L]=v^{i} \frac{\partial L}{\partial v^{i}}-L
$$

is the usual energy of mechanical system, whereas

$$
E[M]=\lambda^{a} \frac{\partial F_{a}}{\partial v^{i}} v^{i}
$$

is the energy of constraints alone.

As seen from (87), in the absence of dissipation, there is a conservation of the total energy; the total means a combination of $E[L]$ and $E[M]$. This conservation law follows also immediately from the Lusternik dynamics.

Indeed, according to the general principles of mechanics, the total energy of our system is:

$$
E[L[\lambda]]=\dot{q}^{i} \frac{\partial L[\lambda]}{\partial \dot{q}^{i}}-L[\lambda]=E[L]+\lambda^{a} \frac{\partial F_{a}}{\partial \dot{q}^{i}} \dot{q}^{i}-\lambda^{a} F_{a}
$$

But on the $F_{a}$-constrained system the last term does vanish and (90) is identical with $E[L, M]$. The additional term $\lambda^{a}\left(\partial F_{a} / \partial v^{i}\right) v^{i}$ describes the exchange of energy between the system of constraints and our object. This is a rather reasonable process when controlling the magnitude of velocity, in any case much more convincing than various XIX-th century procedures based on more or less sophisticated inventions of "virtual displacements". In any case it is so when discussing the modern problems of the active time-dependent control agents.

The problem becomes much more essential when we admit non-holonomic constraints depending also on higher-order time derivatives of generalized coordinates,

$$
F_{A}(q, \dot{q}, \ddot{q}, \ldots, \stackrel{(N)}{q}), \quad A=1, \ldots, m
$$

Obviously, in this expression $\stackrel{(L)}{q}$ denotes the symbol of the $L$-th-order derivatives. The variational Lusternik procedure leads to the following system of equations of motion:

$$
\frac{d}{d t} \frac{\partial L}{\partial \dot{q}^{i}}-\frac{\partial L}{\partial q^{i}}=D_{i}+R_{i}, \quad F_{A}(q, \dot{q}, \ddot{q}, \ldots, \stackrel{(N)}{q})=0
$$

where

$$
R_{i}=\sum_{L=0}^{N}(-1)^{L} \frac{d^{L}}{d t^{L}}\left(\lambda^{A}(t) \frac{\partial F_{A}}{\left.\partial^{(L)}\right)^{i}}\right) .
$$

It is a more academic, nevertheless also interesting problem what are the equations of controlled motion of the system the uncontrolled Lagrangian of which depends on higher-order derivatives, i.e., if it has the general shape:

$$
L(q, \dot{q}, \ddot{q}, \ldots, \stackrel{(M)}{q})
$$

with the same meaning of symbols as in (92). Obviously, at least formally the Lusternik procedure with constraints (91) is still formally applicable and leads to the following equations of motion:

$$
\sum_{K=0}^{N}(-1)^{K+1} \frac{d^{K}}{d t^{K}} \frac{\partial L}{\partial \stackrel{(K)^{i}}{q}}=R_{i}
$$


again with $R_{i}$ given by (93). Principally in a similar way we can discuss controlling constraints given by nondifferential expressions, e.g., integral or other, more general. This will be a subject of the next paper. For some reasons maximally interesting is the special case $N=2$, or also $N \geq 2$. Then the reaction forces are given by

$$
R_{i}=\mu^{a} \frac{\partial F_{a}}{\partial q^{i}}-\frac{D}{D t}\left(\mu^{a} \frac{\partial F_{a}}{\partial \dot{q}^{i}}\right)+\frac{D}{D t}\left(\mu^{a} \frac{\partial F_{a}}{\partial \ddot{q}^{i}}\right)
$$

where the symbol $\frac{D}{D t}$ denotes the total derivative with respect to the variable $t$ occurying in all arguments of functions $F_{a}$. The point is that the second derivatives of of $q^{i}$ with respect to $t$ are very important here. But in the "usual", i.e., constraints-free theory those acceleration variables decideabout the structure of equations of motion. Therefore, for $N \geq 2$ the dependence on acceleration variables becomes much more complicated. Some kind of exception occurs only when $F_{a-s}$ are linear in second derivatives $\ddot{q}^{i}$. Then again $R_{i}$-s do not modify essentially the structure of second order differential equations for $q^{i}(t)$.

\section{Models of rotation-less motion}

There is some interesting question concerning non-holonomic constraints imposed on the affine motion 12. By this we mean the strange and interesting problem of rotation-less motion. We describe this motion in the way that the affine velocity $\Omega^{i}{ }_{j}$ is $g$-symmetric.

$$
\Omega^{i}{ }_{j}-\Omega_{j}{ }^{i}=\Omega^{i}{ }_{j}-g_{j k} g^{i l} \Omega_{l}^{k}=0 .
$$

It is well known that symmetric matrices do not form a Lie algebra. Moreover they induce non-holonomic constraints and they are not integrable to any sub-manifold.

Let us remind that the affine motion is defined by:

$$
\xi^{i}(t)=r^{i}(t)+\varphi_{A}^{i}(t) a^{A}
$$

where $\xi^{i}$ are Euler coordinates and $a^{A}$ are Lagrange coordinates [4]-[57]. The affine velocity is given as follows:

$$
\Omega_{j}^{i}:=\frac{d \varphi^{i} A}{d t}\left(\varphi^{-1}\right)_{j}^{A}
$$

and its co-moving description:

$$
\widehat{\Omega}_{B}^{A}=\left(\varphi^{-1}\right)^{A}{ }_{i} \frac{d \varphi_{B}^{i}}{d t}=\left(\varphi^{-1}\right)^{A}{ }_{i} \Omega^{i}{ }_{j} \varphi^{j}{ }_{B}
$$

We use the polar decomposition of $\varphi$ :

$$
\varphi=U A, \quad \eta_{A B}=g_{i j} U_{A}^{i} U_{B}^{j}, \quad \eta_{A C} A_{B}^{C}=\eta_{B C} A^{C}{ }_{A},
$$

where $U$ are orthogonal (isometric) matrices, i.e., $U \in O(U, \eta ; V, g)$, and $A$ are $\eta$-symmetric, i.e., $A \in \operatorname{Symm}(U, \eta)$, and positively definite. The co-moving angular velocity $\widehat{\omega}$ of the $U$-rotator is as follows:

$$
\widehat{\omega}=U^{-1} \frac{d U}{d t}
$$

of course $\widehat{\omega}$ is $\eta$-skew-symmetric:

$$
\eta_{A C} \widehat{\omega}_{B}^{C}=-\eta_{B C} \widehat{\omega}^{C}{ }_{A}
$$

The kinetic energy is given in the form:

$$
T=T_{t r}+T_{i n t}=\frac{m}{2} g_{i j} \frac{d r^{i}}{d t} \frac{d r^{j}}{d t}+\frac{1}{2} g_{i j} \frac{d \varphi_{A}^{i}}{d t} \frac{d \varphi^{j} B}{d t} J^{A B},
$$

where $m$ and $J^{A B}$ are the total mass and co-moving tensor of inertia respectively given as follows:

$$
m=\int d \mu(a), \quad J^{A B}=\int a^{A} a^{B} d \mu(a) .
$$

In polar decomposition (100) the internal kinetic energy $T_{i n t}$ becomes

$$
\begin{aligned}
T_{i n t} & =\frac{1}{2} \eta_{K L} \frac{d A^{K}{ }_{A}}{d t} \frac{d A_{B}^{L}}{d t} J^{A B}+\eta_{K L} \widehat{\omega}^{K}{ }_{C} A^{C}{ }_{A} \frac{d A_{B}^{L}}{d t} J^{A B} \\
& +\frac{1}{2} \eta_{K L} \widehat{\omega}^{K}{ }_{C} \widehat{\omega}^{L}{ }_{D} A^{C}{ }_{A} A^{D}{ }_{B} J^{A B} .
\end{aligned}
$$


The symmetry constraints on $\Omega$ imply that:

$$
\widehat{\omega}=\frac{1}{2}\left[A^{-1}, \frac{d A}{d t}\right]=\frac{1}{2}\left(A^{-1} \frac{d A}{d t}-\frac{d A}{d t} A^{-1}\right) .
$$

Substituting this to $T_{\text {int }}$ we obtain the simplest vakonomic Lagrangian:

$$
\begin{aligned}
L^{v a k}=T_{i n t}^{v a k}-\mathcal{V}(G) & =\frac{1}{8} \eta_{K L} \frac{d A^{K}{ }_{A}}{d t} \frac{d A^{L}{ }_{B}}{d t} J^{A B} \\
& +\frac{1}{4} \eta_{K L} A^{-1}{ }_{D} \frac{d A^{D}{ }_{C}}{d t} A^{C}{ }_{A} \frac{d A^{L_{B}}}{d t} J^{A B} \\
& +\frac{1}{8} \eta_{K L} A^{-1}{ }_{E} \frac{d A_{C}^{E}}{d t} A_{A}^{C} A^{-1}{ }_{F} \frac{d A_{D}^{F}}{d t} A_{B}^{D} J^{A B}-\mathcal{V}(G),
\end{aligned}
$$

where $\mathcal{V}(G)$ is the potential depending only on the Green deformation tensor:

$$
G_{A B}=g_{i j} \varphi_{A}^{i} \varphi_{B}^{j}=\eta_{C D} A^{C}{ }_{A} A^{D}{ }_{B} .
$$

The above Lagrangian is expressed through $(A, d A / d t)$. We can subject it to the variational procedure by the direct substitution: $A \rightarrow A+\delta A$ and developing the resulting $\delta L$ up to first-order terms in the $\eta$-symmetric $\delta A$.

The structures of vakonomic and d'Alembert equations are different. Details will be disscussed in a forthcoming paper. Obviously, the general d'Alembert Lagrangian has the form:

$$
L=T_{\text {int }}-\mathcal{V}(G)
$$

where $T_{\text {int }}$ is given by (105) and the constraints (106) are eliminated through the d'Alembert procedure of the ideal reactions. Roughly speaking, Lagrangian equations of motion for the "d'Alembert-constrained" system are more complicated and given by the symmetric part of equation of motion without constraints [13]. What is incomparatively more essential, the d'Alembert equations of motion are obtained in different way: firstly one takes the symmetric part of the tensorial equations of motion and only then one substitutes to them the condition $\Omega^{i}{ }_{j}=\Omega_{j}{ }^{i}$. In vakonomic theory the sequence of procedure is reversed: first one substitutes the symmetry condition to the Lagrangian, only then one performs the variation with respect to the symmetric $\Omega^{i j}$. The two procedures are non-commutative. This is evidently something else than the procedure obtained from (107) by the method described after it, just the special case of the difference between (36) and (52)

The vakonomic model from mathematical point of view seems to be more elegant, but it is hard to say that it is better in usual non-holonomic problems of the slide-free motion. However, it is still being a promising procedure, which is applicable in many areas (e.g., finance, robotics, control, biological and nuclear problems). In general they seem to be useful for systems with higher disorder.

\section{Acknowledgements}

This paper partially contains results obtained within the framework of the research project N N501 049540 financed from the Scientific Research Support Fund in the years 2011-2014. The authors are greatly indebted to the Polish Ministry of Science and Higher Education for this financial support. I'm greatful to Proffesor J. J. Sławianowski for consultations concerning constreints, and couraging me to get into this topic.

\section{References}

[1] R. Abraham and J.E. Marsden, Foundations of Mechanics, Benjamin-Cummings, (1978).

[2] V. I. Arnold, V. V. Kozlov, and A. I. Neishtadt, Mathematical Aspects of Classical and Celestial Mechanics, Springer, New York, 1997.

[3] R. Benito and D. M. de Diego, Discrete Vakonomic Mechanics, Math. Phys. 46, 083521 (2005); http://dx.doi.org/10.1063/1.2008214 (18 pages).

[4] A. Burov and D. P. Chevallier, On the Variational Principle of Poincare, the Poincare-Chetayev Equations and the Dynamics of Affinely Deformable Bodies, Cahier de C.E.R.M.I.C.S. 14, Mai 1996.

[5] G. Capriz, Continua with Microstructure, Springer Tracts in Natural Philosophy 35, Springer-Verlag, New York-Berlin-Heidelberg-Paris-Tokyo, 1989.

[6] D. P. Chevallier, On the Foundations of Ordinary and Generalized Rigid Body Dynamics and the Principle of Objectivity, Arch. Mech. 56 (2004), no. 4, 313-353. 
[7] H. Cohen and M. G. Muncaster, The Theory of Pseudo-Rigid Bodies, Springer Tracts in Natural Philosophy, Springer, Berlin, 1989.

[8] J. Cortes, M. de Leon, D. M. de Diego, and S. Martinez, Geometric Description of Vakonomic and Nonholonomic Dynamics. Comparison of Solutions, SIAM J. Control Optim. 41 (2003), no. 5, 1389-1412.

[9] A. C. Eringen, Mechanics of Micromorphic Continua, in: Proceedings of the IUTAM Symposium on Mechanics of Generalized Continua (Freudenstadt and Stuttgart, 1967), E. Kröner (ed.), vol. 18, Springer, Berlin-Heidelberg-New York, 1968, 18-33.

[10] B. Gołubowska, Motion of Test Rigid Bodies in Riemannian Spaces, Rep. on Math. Phys. 48 (2001), no. $1 / 2,95-102$.

[11] B. Gołubowska, Models of Internal Degrees of Freedom Based on Classical Groups and Their Homogeneous Spaces, Rep. on Math. Phys. 49 (2002), no. 2/3, 193-201.

[12] B. Gołubowska, Affine Models of Internal Degrees of Freedom and Their Action-Angle Description, Rep. on Math. Phys. 51 (2003), no. 2/3, 205- 214.

[13] B. Gołubowska, V. Kovalchuk, E. E. Rożko and J. J. Sławianowski: Some Constraints and Symmetries in Dynamics of Homogeneously Deformable Elastic Bodies, in: I. M. Mladenov, A. Ludu and A. Yoshioka (eds), Geometry, Integrability and Quantization XIV, Avangard Prima, Sofia 2013, 138-144.

[14] J. J. Sławianowski, B. Gołubowska, and V. Kovalchuk, Constraints and symmetry in mechanics of affine motion, arXiv: 1302.0674v1

[15] R. Gutowski, Analytical mechanics, PWN, Warszawa, 1971 (in Polish)

[16] M. Jóźwikowski, W. Respondek A comparison of vakonomic and nonholonomic variational problems with applications to systems on Lie groups, arXiv:1310.8528v1 [math.DG], 31 Oct. 2013.

[17] V. Kovalchuk, On Classical Dynamics of Affinely-Rigid Bodies Subject to the Kirchhoff-Love Constraints, SIGMA (Symmetry, Integrability and Geometry: Methods and Applications) 6 (2010), no. 031, 12 pages.

[18] V. Kovalchuk: Green Function for Klein-Gordon-Dirac Equation, J. Nonlin. Math. Phys. 11, Supplement, $72-77(2004)$.

[19] V. V. Kozlov Realization of nonintegrable constraints in classical mechanics, Sov. Phys. Dokl., 1983, vol. 28 , pp. $735-737$.

[20] V. V. Kozlov Dynamics of systems with nonintegrable constraints. V: Freedom principle and ideal constraints condition Mosc. Univ. Mech. Bull., 1988, vol. 43, no. 6, pp. 23-29.

[21] P. M. Mariano, Configuration Forces in Continua with Microstructure, Z. angew. Math. Phys. 51 (2000), $752-791$.

[22] A. Martens and J. J. Sławianowski, Affinely-Rigid Body and Oscillatory Dynamical Models on GL(2, $\mathbb{R})$, Acta Physica Polonica B 41 (2010), no. 8, 1847-1880.

[23] G. W. Mackey, The mathematical foundations of quantum mechanics, Benjamin, 1963.

[24] J. Nejmark, N. Fufajew: Dynamika uktad(́)w nieholonomicznych, PWN, Warszawa, 1981.

[25] O. M. O'Reilly, A Properly Invariant Theory of Infinitesimal Deformations of an Elastic Cosserat Point, Z. angew. Math. Phys. 47 (1996), 179-193.

[26] O. M. O'Reilly and P. C. Varadi, A Unified Treatment of Constraints in the Theory of a Cosserat Point, Z. angew. Math. Phys. 49 (1998), 205-223.

[27] P. Papadopoulos, On a Class of Higher-Order Pseudo-Rigid Bodies, Math. Mech. Solids 6 (2001), 631-640.

[28] M. Roberts, C. Wulff, and J. Lamb, Hamiltonian Systems Near Relative Equilibria, J. of Diff. Equations 179 (2002), 562-604.

[29] E. E. Rożko, Dynamics of Affinely-Rigid Bodies with Degenerate Dimension, Rep. on Math. Phys. 56 (2005), no. 3, 311-332.

[30] E. E. Rożko, Quantization of Affinely-Rigid Bodies with Degenerate Dimension, Rep. on Math. Phys. 65 (2010), no. 1, 1-15. 
[31] M. B. Rubin, On the Theory of a Cosserat Point and Its Application to the Numerical Solution of Continuum Problems, ASME J. Appl. Mech. 52 (1985), 368-372.

[32] M. B. Rubin, Free Vibration of a Rectangular Parallelepiped Using the Theory of a Cosserat Point, ASME J. Appl. Mech. 53 (1986), 45-50.

[33] J. J. Sławianowski, Analytical Mechanics of Finite Homogeneous Strains, Arch. of Mech. 26 (1974), no. 4, $569-587$.

[34] J. J. Sławianowski, The Mechanics of an Affinely-Rigid Body, Int. J. of Theor. Phys. 12 (1975), no. 4, $271-296$.

[35] J. J. Sławianowski, Newtonian Dynamics of Homogeneous Strains, Arch. of Mech. 27 (1975), no. 1, 93-102.

[36] J. J. Stawianowski, The Mechanics of the Homogeneously-Deformable Body. Dynamical Models with High Symmetries, Z. angew. Math. Mech. 62 (1982), 229-240.

[37] J. J. Sławianowski, Analytical Mechanics of Deformable Bodies, PWN - Polish Scientific Publishers, Warszawa-Poznań, 1982 (in Polish).

[38] J. J. Sławianowski, Algorithms for Reactions of Non-Holonomic Constraints and Servo-Constraints, Arch. Mech. 39 (1987), no. 6, 645-662.

[39] J. J. Sławianowski, An Affinely-Rigid Body and Hamiltonian Systems on GL(n, R), Rep. on Math. Phys. 26 (1988), no. 1, 73-119.

[40] J. J. Sławianowski, Geodetic Systems on Linear and Affine Groups. Classics and Quantization, J. of Nonlinear Math. Phys. 11 (2004), Supplement, 130-137.

[41] J. J. Sławianowski, Classical and Quantized Affine Models of Structured Media, Meccanica 40 (2005), no. 4-6, 365-387.

[42] J.J.Sławianowski Geometry of Phase Spaces John Wiley \& Sons, Chichester-New York-Brisbane-TorontoSingapore PWN - Polish Scientific Publishers, Warsaw, 792 pages, 1991.

[43] J. J. Slawianowski and B. Golubowska, Motion of test bodies with internal degrees of freedom in noneuclidean spaces, Rep. on Math. Phys. 65, pp. 379-422, 2010.

[44] J. J. Slawianowski and V. Kovalchuk, Klein-Gordon-Dirac equation: Physical justification and quantization attempts, Rep. on Math. Phys. 49 (2002), 249-257.

[45] J. J. Sławianowski and V. Kovalchuk, Invariant Geodetic Problems on the Affine Group and Related Hamiltonian Systems, Rep. on Math. Phys. 51 (2003), no. 2/3, 371-379.

[46] J. J. Sławianowski and V. Kovalchuk, Classical and Quantized Affine Physics: A Step Towards It, J. of Nonlinear Math. Phys. 11 (2004), Supplement, 157-166.

[47] J. J. Sławianowski, V. Kovalchuk, A. Sławianowska, B. Gołubowska, A. Martens, E. E. Rożko, and Z. J. Zawistowski, Affine Symmetry in Mechanics of Collective and Internal Modes. Part I. Classical Models, Rep. on Math. Phys. 54 (2004), no. 3, 373-427.

[48] J. J. Sławianowski, V. Kovalchuk, A. Sławianowska, B. Gołubowska, A. Martens, E. E. Rożko, and Z. J. Zawistowski, Affine Symmetry in Mechanics of Collective and Internal Modes. Part II. Quantum Models, Rep. on Math. Phys. 55 (2005), no. 1, 1-45.

[49] J. J. Sławianowski, V. Kovalchuk, B. Gołubowska, A. Martens, and E. E. Rożko, Quantized Excitations of Internal Affine Modes and Their Influence on Raman Spectra, Acta Physica Polonica B 41 (2010), no. 1, $165-218$.

[50] J. J. Sławianowski, V. Kovalchuk, A. Martens, B. Gołubowska, and E. E. Rożko, Mechanics of Systems of Affine Bodies. Geometric Foundations and Applications in Dynamical of Structured Media, Mathematical Methods in the Applied Sciences 34 (2011), no. 12, 1512-1540.

[51] J. J. Sławianowski, V. Kovalchuk, A. Martens, B. Gołubowska, and E. E. Rożko, Essential Nonlinearity Implied by Symmetry Group. Problems of Affine Invariance in Mechanics and Physics, Discrete and Continuous Dynamical Systems — Series B 17 (2012), no. 2, 699-733.

[52] J. J. Sławianowski and A. K. Sławianowska, Virial Coefficients, Collective Modes and Problems with the Galerkin Procedure, Archives of Mechanics 45 (1993), no. 3, 305-331. 
[53] J. M. Solberg and P. Papadopoulos, A Simple Finite Element-Based Framework for the Analysis of Elastic Pseudo-Rigid Bodies, Int. J. Numer. Meth. Eng. 45 (1999), 1297-1314.

[54] J. M. Solberg and P. Papadopoulos, Impact of an Elastic Pseudo-Rigid Body on a Rigid Foundation, Int. J. Eng. Sci. 38 (2000), 589-603.

[55] E. Sousa Dias, A Geometric Hamiltonian Approach to the Affine Rigid Body, in: Dynamics, Bifurcation and Symmetry. New Trends and New Tools, P. Chossat (ed.), NATO ASI Series C 437, Kluwer Academic Publishers, Netherlands, 1994, 291-299.

[56] A. Trzȩsowski and J. J. Sławianowski, Global Invariance and Lie-Algebraic Description in the Theory of Dislocations, Int. J. of Theor. Phys. 29 (1990), no. 11, 1239-1249.

[57] C. Wulff and M. Roberts, Hamiltonian Systems Near Relative Periodic Orbits, SIAM J. of Dynamical Systems 1 (2002), no. 1, 1-43. 